\title{
Exploring The Molecular Interaction of Eugenol Targeting Main Protease of SARS Cov2 Virus Through In-silico Studies
}

\author{
Heenu Dhar and Poonam Salwan \\ Department of Pharmacology Shree Guru Gobind Singh Tricentary \\ University, Gurugram, Haryana, India \\ Corresponding author email: heenu_fmhs@sgtuniversity.org
}

\section{ABSTRACT}

Since the outbreak of COVID-19 millions of people have been affected and thousands of people are dead. This infectious disease have propagated through a new virus strain as SARS CoV2 (Sever Acute Respiratory Syndrome Coronavirus 2). Natural compounds are always helping the humans to overcome many viral disease. Here, in this research an attempt was made to discover and help researchers to find the better phyto-chemical that can act as an inhibitor to treat the patients. Eugenol (Clove Oil) have been used to treat many viral and against many anti-cancer diseases. Molecular docking performed on Eugenol showed effective interaction with the Main Protease of SARS CoV2. Bonds and Distance between bonds also verified that Eugenol can be the candidate to develop an anti-viral vaccine or drug. Although further research is needed on this topic to overcome this pandemic.

KEY WORDS: ATOMIC CONTACT ENERGY (ACE), COVID-19, EUGENOL, GLOBAL ENERGY, SARS COV2, WORLD HEALTH ORGANISATION (WHO).

\section{INTRODUCTION}

As this research is in progress, cases of COVID-19 (CoronaVirus Disease) are increasing at a rapid rate. Today, on October 20th 2020, 41M humans (Figure 1) have been affected by the deadly coronavirus as reported by the World Health Organization (WHO). Globally $30 \mathrm{M}$ cases have been recovered while a large population of $1 \mathrm{M}$ cases is dead and $10 \mathrm{M}$ cases are still active. The United State of America is on the top with $8.5 \mathrm{M}$ cases followed by India with $7.5 \mathrm{M}$ cases(Worldometer et. al. (2020)).

Coronaviruses belong to the family Coronaviridae, these diseases, if left untreated, can cause many deadly outcomes to many important organs of our body like respiratory, enteric,

Biosc Biotech Res Comm P-ISSN: 0974-6455 E-ISSN: 2321-4007
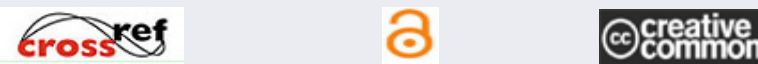

Identifiers and Pagination

Article Information

Year: 2021 Vol: 14 No (6) Special Issue

Pages: 366- 369

Received: $19^{\text {th }}$ May 2021

This is an open access article under Creative

Commons License Attribn 4.0 Intl (CC-BY).

DOI: $h t t p: / / d x . d o i . o r g / 10.21786 / b b r c / 14.7 .77$ or neurological systems. Among all the coronaviruses, Severe Acute Respiratory SyndromeCoronavirus-2 (SARS $\mathrm{CoV} 2$ ) has arose as a pandemic to human society with a mortality rate of $3-4 \%$. SARS CoV2 is positive sense, single stranded RNA virus of approximately $30 \mathrm{~kb}$. As per history and genetic similarities, it is said that bats are the hosts in nature which contains SARS CoV2(U. Umesh et. al. (2020).

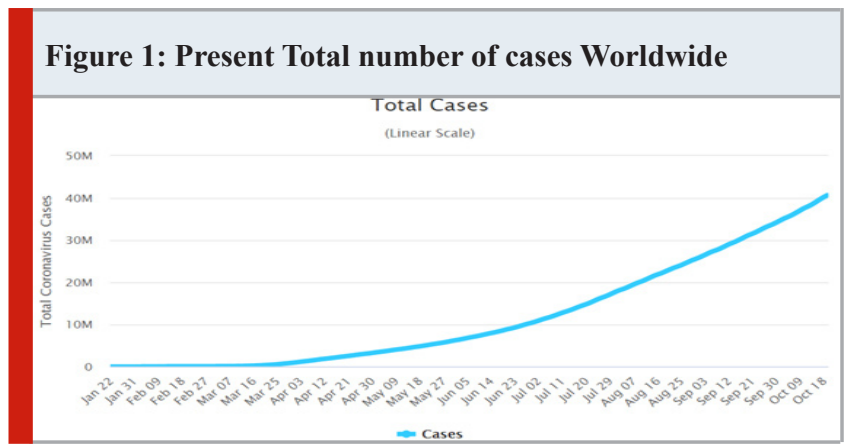

SARS CoV2 consists of four proteins namely Spike (S), Membrane (M), Envelope (E) and Nucleoplasid (N). These 
four structural proteins are responsible for the recognition of a receptor on the host cell fusing the virus with the host membrane receptor and further transmitting the infection within the host. The spike protein of Virus fuses with the ACE2 receptor of the host cell, drug can be developed by hindering this fusion. But patients that are already infected, the target is main protease (MPro). MPro or 3CLPro is a dimer that have six strands of anti-parallel $\beta$ barrels, the substrate binding site is in the middle and along with the site there is a group of five helices which holds the function of dimerization of the enzyme(R. J. Khan (2020), P. Shree (2020)). MPro is targeted because it is involved in the translation of viral RNA into the polyproteins that disturbs the normal functioning of a human and forms the viral RNA polymerase, endoribonuclease and exoribonuclease. Antiviral like Lopinavir, Ritonavir, Nelfinavir and Remdesivir and their combination have been striked as therapeutics to counter the coronavirus. Present study shows the importance of phytoconstituent targeting the SARS CoV2 Main Protease(P. Shree (2020, Q. Zou (2020).

Based on health, Nature and life, natural products and their constituents have always nourished the human body. There are many medicinal plants that contain the key to treat many life threatening diseases. Nature holds a wide range of medicinal plants that can cure respiratory disorders, one of them is Syzygiumaromaticum(Clove) used in current study. Major constituent of Clove is Eugenol which constitutes about $80 \%-90 \%$ of the clove. Eugenol is a phenol which is very less soluble in water but it is easily soluble in organic solvents. It has been effective against many viral diseases like Ebola virus, anti-influenza virus A, Herpes and Antiherpes virus and many more. Natural products formulations can play a notable role to develop a therapeutic drug that not onlt be safe but will be non-toxic to the human body(P. Shree (2020), A. H. Kumar (2020), P. Ardra).

Literature Review: A research conducted on anethole, cinnamaldehyde, carvacrol, geraniol, cinnamyl acetate, L-4terpineol, thymol and pulegone as Lamiaceae, Lauraceae, Myrtaceae, Apiaceae, Geraniaceae and Fabaceae to analyse the potential of aforementioned phyto-compounds on Main-protease SARS CoV2. In-silicostudies concluded that aformentionedphyto-compounds are the are effective antiviral agents that have potential to inhibit the viral S1 receptor binding site of spike protein(S. A. Kulkarni et. al. (2020).

Another research conducted on bioactive compounds found in plants that can be the inhibitors of the Main Protease and Spike Protein of SARS CoV2. In-silicostudies were conducted on many bioactive natural compounds but hesperidin, nabiximols, pectolinarin,epigallocatechin gallate, and rhoifolin were found to be the ones that have the best binding energies with the MPro and S-protein of SARs CoV2, there it was concluded that these are the potent inhibitor of COVID-19(T. E. Tallei et. al. (2020). In-silicoresearch conducted onayurvedic medicinal plants likeAshwagandha, Tulsi and Giloy on the Main protease of SARS CoV2. It was concluded that aforementioned ayurvedicplants have a variety of active phyto-compounds that are the inhibitor of SARS CoV2 and can be used to design a drug or vaccine against COVID-19 (P. Shree et. al. (2020)).

A research conducted on tulsi and neem against 3 main proteins namely Surface Glycoprotein, RNA Dependent RNA polymerase and Main of SARS CoV2discussed the docking studies. Chimera results revealed that neem and tulsi have a great quantity of active phyto-compounds that can be the potent inhibitor of aforementioned viral proteins. (A. H. Kumar et. al. (2020). There is very little research done on the in-silico studies of Eugenol on SARS CoV2 Main protease. Current research will discuss the molecular interactions between the Eugenol on SARS CoV2 Main protease.

Research Questions: What are the effects of Eugenol on SARS CoV2 Main protease?

\section{METHODOLOGY}

Design: As Shown in Figure 2, PDB formatted files of $3 \mathrm{D}$ structure of ligand which is Eeugenolwas downloaded from PubChem and 3D Quaternary structure of Receptor i.e., SARS CoV2 Main Protease was downloaded from RCSB PDB. After downloading these files are formatted to a same file format as .pdb and then these two files are used for docking. To dock these structure online tools are used i.e. PatchDock and Fire Dock. After obtaining the docking results the docked complex was visualised using the BOVIA drug discovery studio and PyMOL.
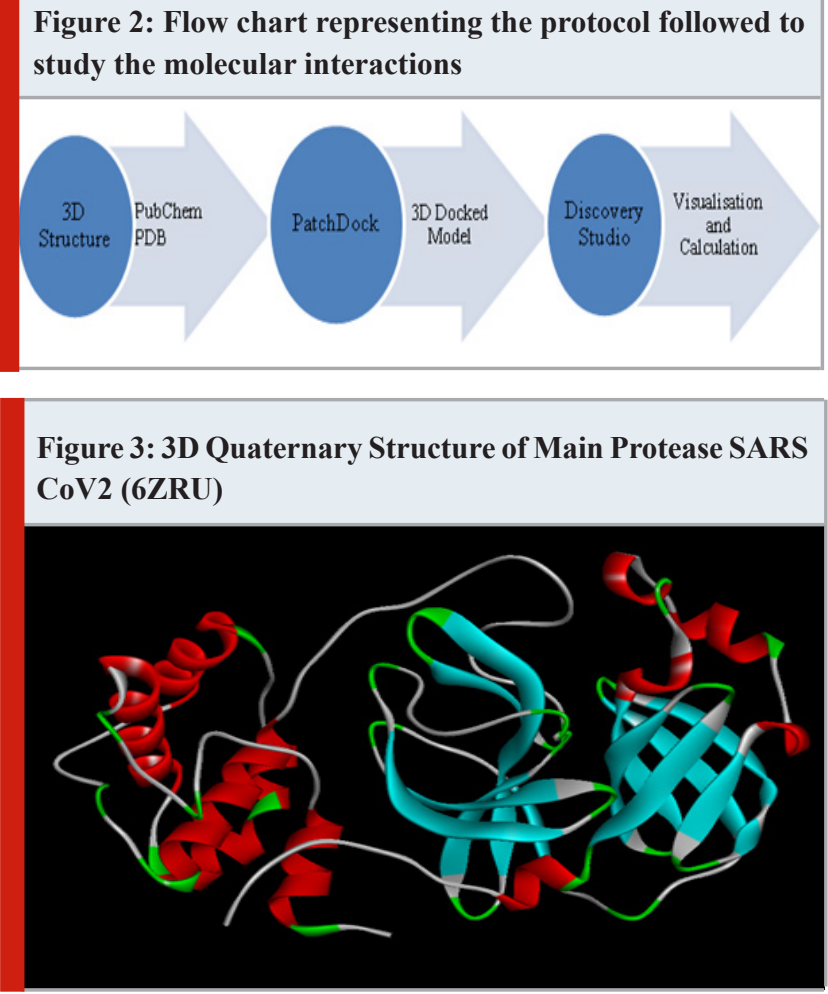

Sample: 3D Quaternary Structure of Main Protease SARS CoV2 under the ID of 6ZRU (Figure 3) was downloaded from RCSB PDB and 3D structure of Eugenol (Figure 4) was downloaded from PubChem in SDF format. In Figure 
2 , red spirals are representing the $\alpha$-helices while the cyan coloured ribbons are the $\beta$ plated sheets. In Figure 3 , Green balls are representing the carbon atoms, red balls are representing the oxygen atom and the white balls are for the hydrogen atom. These structure are further used for docking studies.

Figure 4: 3D ball and stick Structure of Eugenol

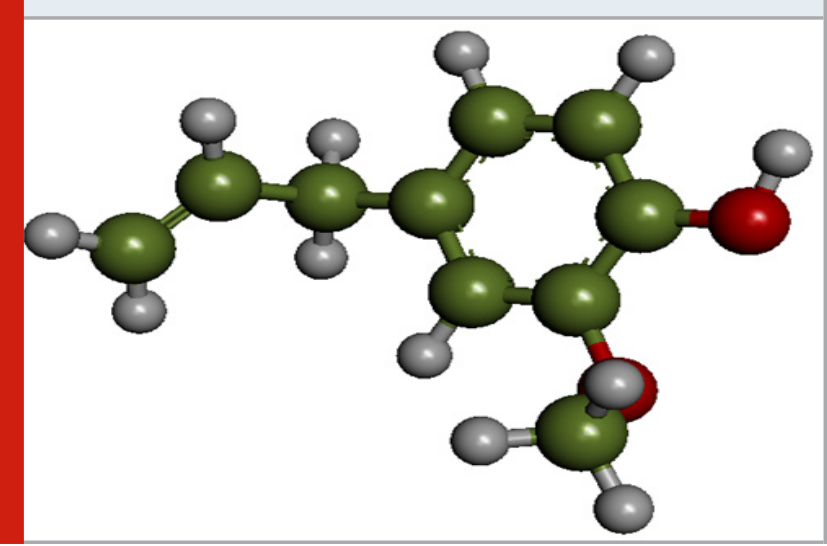

Instrument: File containing the 3D structure of Eugenol was downloaded from Public Chemistry (PubChem). It is launched in 2004 by National institute of Health (NIH). It is an open database full of chemical information, open means that anyone can put the data any it can be used by anyone. PubChem consists of major chemical information lice chemical properties, chemical 2D or 3D structures, patents, toxicity information and many other relevant information that is used by many researchers to complete their research and further help the world. File containing the 3D structure of SARS CoV2 Main Proteeaase was downloaded from RCSB PDB. It is an open database led by Helen M. Berman. Research Collaborators for Structural Bioinformatics (RCSB) Protein Data Bank (PDB), here all the information about the different viral, bacterial and any other protein information can be found and can be downloaded. This information is used by many researchers to complete their research and further help the world.

PATCHDOCKis a free available web server that is used for molecular docking, .pdb formatted files of ligand and receptor were uploaded to this server. It is a docking algorithm that performs docking on the basics of geometry. It finds the docking transformations that give the best complementarity. It is highly efficient because of its fast transformational search. After Pathdock, use firedock. Firedock gives the Global energy of the docked models. It is an open and a branched server of PatchDock. With the help of BOVIA Drug Discovery Studio docked complex were visualised, It is a molecular modelling suite that has various features that helps in molecular modelling and simulation.

Data Collection: After uploading the .pdb formatted files in PatchDock server, different files regarding the docked complex were obtained. Top 10 docked complex as shown in Table 1 was selected for further analysis on the basis of their global energy and ACE (Atomic contact Energy). The complex with the least ACE and Global energy was selected. Selected 3D docked complex was downloaded from FireDock and visualised in BOVIA Drug Discovery Studio. Further, ligand - receptor interactions were constructed and the amino acids that were not forming the bonds with the ligand were removed as shown in Figure 5.

\begin{tabular}{|c|c|c|c|}
\hline S.No. & Score & $\begin{array}{c}\text { Global Energy } \\
\text { (kcal/mol) }\end{array}$ & $\begin{array}{c}\mathrm{ACE} \\
(\mathrm{kcal} / \mathrm{mol})\end{array}$ \\
\hline 1 & 3256 & -25.55 & -104.4 \\
\hline 2 & 3176 & -21.11 & -105.97 \\
\hline 3 & 3122 & -21.86 & -47.83 \\
\hline 4 & 3086 & -21.10 & -75.06 \\
\hline 5 & 3026 & -16.72 & -14.88 \\
\hline 6 & 3010 & -24.53 & -124.14 \\
\hline 8 & 3010 & -19.52 & -100.58 \\
\hline 7 & 2950 & -19.11 & -135.01 \\
\hline 9 & 2878 & -17.44 & -17.99 \\
\hline 10 & 2864 & -29.06 & -123.17 \\
\hline
\end{tabular}

Figure 5: 3D Structure of Docked model 1 showing Amino Acids

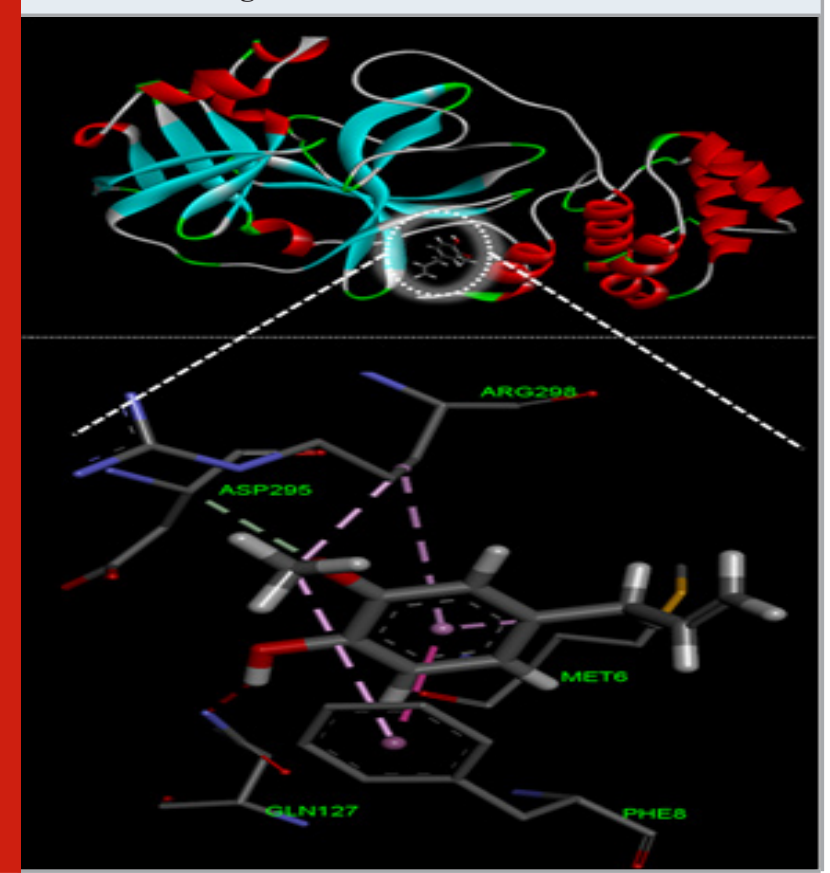

Data Analysis: As shown in Table 2, 6 bonds were formed with Hydrophobic and hydrogen bonds between the ligand which is Eugenol and receptor which is SARS CoV2 Main Protease. Atomic Contact Energy or ACE is the energy of replacing an atom/molecule with another atom or molecule whereas Global Energy or Gibbs free energy is same as the binding energy of ligand and receptor. The least the energy the more efficient the complex. 
Table 2. Bonds formed, Distance and Bond Type between Eugenol and $M^{\text {Pro }}$

\begin{tabular}{|c|c|c|c|}
\hline S.No. & $\begin{array}{c}\text { Ligand : } \\
\text { Protein }\end{array}$ & Distance & $\begin{array}{c}\text { Bond } \\
\text { Type }\end{array}$ \\
\hline 1. & UNL1 : ASP295 & 3.79759 & Hydrogen Bond \\
\hline 2. & UNL1 : PHE8 & 3.95976 & Hydrophobic \\
\hline 3. & UNL1 : ARG298 & 3.18874 & Hydrophobic \\
\hline 4. & UNL1 : PHE8 & 4.79218 & Hydrophobic \\
\hline 5. & UNL1 : MET6 & 5.43217 & Hydrophobic \\
\hline 6. & UNL1 : ARG298 & 4.71877 & Hydrophobic \\
\hline
\end{tabular}

\section{RESULTS AND DISCUSSION}

In-Silico studies are the first step towards the knowledge that whether the compound is interacting with the disease protein or not. They draw a probable picture which can be further used to do the in-vitro studies. In this present research the in-silico studies were performed on Eugenol and Main protease of SARS CoV2 and it was found out that the Eugenol was interacting with the MPro. Top 10 docked models were evaluated and the Model 1 was selected because of highest score of 3256. Global Energy which is also the binding energy of model 1 was $-25.55 \mathrm{kcal} /$ mol and the Atomic Contact Energy (ACE) was found out to be $-104.44 \mathrm{kcal} / \mathrm{mol}$. Distance between the bonds we ranging from 3-5.5 and one hydrogen and 5 hydrophobic bonds were forming between the Eugenol and the MPro SARS CoV2.

\section{CONCLUSION}

COVID-19 is a viral respiratory diseases that is spreading at a rapid rate and affecting a large amount of people worldwide. This disease needs to be controlled and it should be prevent from further spreading. Eugenol, a phyto-chemical, can act as a potent antiviral agent against this disease. This study plays a vital role in identifying the role of Eugenol to overcome the problems occurring in preparation and finding a anti-viral Results revealed that Eugenol have an efficient ACE and binding energy and it can be a new therapeutic that needs to be researched upon. Further in-slico and in-vitro studies are required to affirm the eugenol efficacy towards SARS CoV2.

\section{REFERENCES}

Chowdhury, P., 2020. In silico investigation of phytoconstituents from Indian medicinal herb 'Tinospora cordifolia (giloy)'against SARS-CoV-2 (COVID-19) by molecular dynamics approach. Journal of Biomolecular Structure and Dynamics, pp.1-18.

COVID, W., 19. Coronavirus Pandemic: Coronavirus Cases.

Kumar, A.H., 2020. Molecular docking of natural compounds from tulsi (Ocimum sanctum) and neem (Azadirachta indica) against SARS-CoV-2 protein targets. Biology, Engineering, Medicine and Science Reports, 6(1), pp.11-13.

Shree, P., Mishra, P., Selvaraj, C., Singh, S.K., Chaube, R., Garg, N. and Tripathi, Y.B., 2020. Targeting COVID19 (SARS-CoV-2) main protease through active phytochemicals of ayurvedic medicinal plants-Withania somnifera (Ashwagandha), Tinospora cordifolia (Giloy) and Ocimum sanctum (Tulsi)-a molecular docking study. Journal of Biomolecular Structure and Dynamics, pp.114.

Singh, P., Hariprasad, V.R., Babu, U.V., Rafiq, M. and Rao, R.P., 2020. Potential phytochemical inhibitors of the coronavirus RNA dependent RNA polymerase: a molecular docking study.

Tallei, T.E., Tumilaar, S.G., Niode, N.J., Kepel, B.J., Idroes, R., Effendi, Y., Sakib, S.A. and Emran, T.B., 2020. Potential of plant bioactive compounds as SARSCoV-2 main protease (Mpro) and spike (S) glycoprotein inhibitors: a molecular docking study. Scientifica, 2020. Umesh, Kundu, D., Selvaraj, C., Singh, S.K. and Dubey, V.K., 2021. Identification of new anti-nCoV drug chemical compounds from Indian spices exploiting SARS-CoV-2 main protease as target. Journal of Biomolecular Structure and Dynamics, 39(9), pp.3428-3434.

Vickers, N.J., 2017. Animal communication: when i'm calling you, will you answer too?. Current biology, 27(14), pp.R713-R715.

Wheeler, D.L., 2003. Egypt: Building an information society for international development. Review of African Political Economy, 30(98), pp.627-642. 